\title{
Mobile Station Position Estimation using Remote Radio Head with Adaptive Power Control
}

\author{
Hyeon-Cheol Lee, Sang Gyu Lee and Seung Hoon Lee \\ Payload Electronics Team, Satellite Payload Research Office, Satellite R\&D Head Office, Korea Aerospace Research Institute, \\ 169-84, Gwahak-ro, Yuseong-gu, Daejeon 34133, Rep. of Korea
}

\begin{abstract}
The adaptive power control of CDMA (Code Division Multiple Access) communications between multiple MSs (Mobile Stations) with a link-budget based SIR (Signal-to-Interference Ratio) estimate is applied to inner loop power control algorithms. CTR (Consecutive Transmit-Power-Control Ratio) calculated from these algorithms can estimate MS speed, together with MS moving distance, but cannot estimate MS position. In this paper, RRH (Remote Radio Head) is introduced and it is concluded that BS (Base Station) calculates MS distance with CTR from one of RRHs, in addition BS that has RSSIs (Received Signal Strength Indicators) information from other two RRHs can estimate MS position.
\end{abstract}

Key words: Position estimation, adaptive power control, link-budget, SIR, RRH.

\section{Introduction}

MSs (Multiple Mobile Stations) need mobile wireless network to share data between MSs and a possible network is CDMA (Code Division Multiple Access). CDMA different from TDMA and FDMA uses same frequency for multiple users. Since all users utilize a single frequency, the near-far effect is generated [1]. To eliminate the near-far effect in CDMA systems, the transmission signal power from every MS must be the same level at the receiver. This technique of controlling the magnitude of the transmission power according to distance between MS and BS (Base Station) is officially termed power control. It equalizes the received power and eliminates the near-far effect, though it is subject to such complications as path loss, shadowing, multi-path fading, etc. It is called adaptive power control when adaptive algorithm is applied to update RF power.

This power control technique is differentiated into open loop power control and closed loop power control. The closed loop power control is further divided into inner loop power control and outer loop

Corresponding author: Hyeon-Cheol Lee, Ph.D., principal researcher, research fields: communications, SAR. power control. The inner loop power control is responsible for adjusting the power transmitted to maintain the received SIR (Signal-to-Interference Ratio) at BS at a level equal to that at the $\operatorname{SIR}_{\text {target }}$. The outer loop power control is responsible for setting the SIR $_{\text {target }}$ based on the BER (Bit Error Rate) or service requirement.

In general, an aircraft measures its air speed by pitot tube and calculates its ground speed by GPS/INS (Global Position system/Inertial Navigation System). In these days, there is an effort that optic flow measurements from CCD camera have been used to augment GPS/INS to provide more precise velocity information [2]. GPS/INS which provides speed, position and attitude information, however has errors when GPS is not available or cannot provide exact information when communication system is not robust.

Measuring speed by SIR estimate is attempted when other speed measurements are not available. Conventional SIR estimates [3,4] consider only the transmission power and the link-gain, and a CTR (Consecutive Transmit-Power-Control Ratio) is introduced for speed estimation in Ref. [5] because a faster mobile is likely to receive more CTR than a 
slower one. The paper [6] takes into account the link-budget, which has more realistic parameters including distance information and path loss than the link-gain and shows more exact relationship between speed and CTR.

BS which has one antenna estimates MS speed only, however, it cannot provide MS position information. This paper introduces MS distance and position estimation using RRH (Remote Radio Head) [7] on the basis of speed estimation of adaptive power control. In Fig. 1, BS that installs three RRHs calculates MS distance with CTR from RRH-A, and BS calculates MS distance with the RSSI (Received Signal Strength Indicator) ratio from RRH-B and RRH-C which measure received signal strength from MS.

This paper is organized as follows: The literature related to this work is surveyed in Section 2. The inner loop power control is described in Section 3. The review of the link-budget based SIR estimate is introduced in Section 4, followed by description of simulation environments in Section 5. The simulation results are analyzed in Section 6. Finally, conclusions are drawn in Section 7.

\section{Related Work}

Kim et al. [8] introduced in the AS-CLPC (Adaptive Step-size Closed Loop Power Control) algorithm for a narrowband CDMA system. This algorithm adapts its power control step-size based on the optimal factors determined with the mean fade duration which is inversely proportional to the maximum doppler frequency. Nourizadeh, Taaghol, and Tafazolli [9] proposed the BA-CLPC (Blind Adaptive Closed Loop Power Control) in which the power control step-size is adjusted to cope with the user mobility. Taaghol [10] introduced the SA-CLPC (Speed Adaptive Closed Loop Power Control) algorithm in which the power control step-size is selected based on the user speed estimation categorized by speed ranges. Lee and Cho [11] proposed the M-ACLPC (Mobility Based Adaptive Closed Loop Power Control) algorithm in which the power control step-size is adjusted depending on the combination of the cumulative information of the three power control commands and speed estimation.

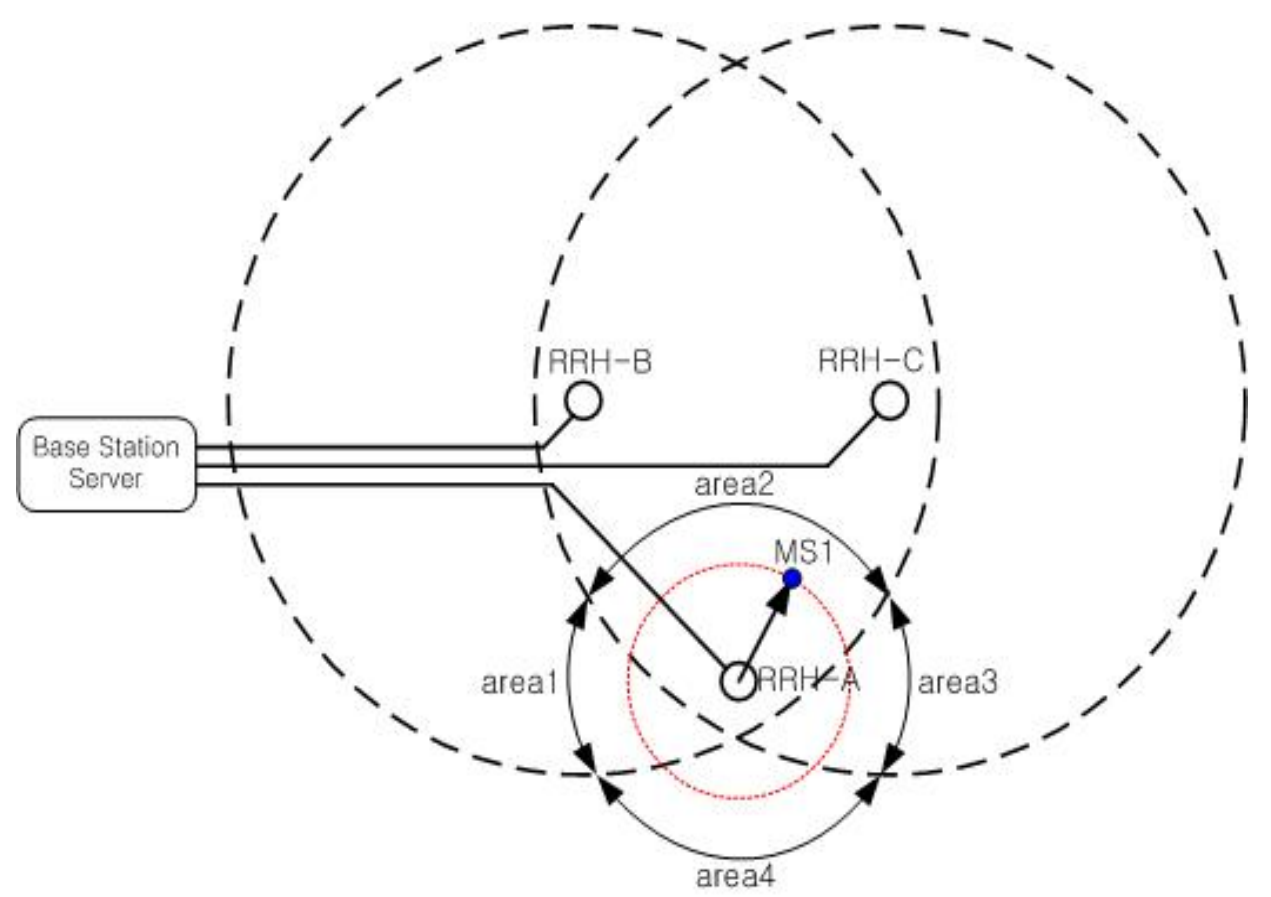

Fig. 1 BS connecting Remote Radio Heads (- - -(black) : RF radiation boundary of RRH antenna, ...(red) : MS1 distance calculated by CTR). 
Patachaianand and Sandrasegaran [4] compared performances of the AS-CLPC, BA-CLPC, SA-CLPC, and M-ACLPC in terms of PCE (Power Control Error) under the same simulation environment. In their comparisons, the AS-CLPC showed the best performance when the MS speed was lower than 25 $\mathrm{km} / \mathrm{h}$, while the SA-CLPC was the best when the speed was greater than $25 \mathrm{~km} / \mathrm{h}$.

Patachaianand and Sandrasegaran [4] presented the CS-CLPC (Consecutive TPC Ratio (CTR) Step-size Closed Loop Power Control) algorithm whose power control step-size is determined based on a parameter called CTR. They measured the moving MS speed by CTR, then, calculated the PCE as the RMS (Root Mean Square) of the difference between the received SIR and the SIR target. They also suggested in Ref. [5] the mapping equation and mapping table which can yield accurate speed estimation using CTR.

Lee [6] presents MS speed estimation with multi-bit quantizer in Adaptive Power Control. The algorithm shows linearity between speed and CTR of CS-CLPC, and FSPC (Fixed Size Power Control), but CTR of AS-CLPC is independent of its speed. With this linearity, MS moving distance can also be calculated.

\section{Inner Loop Power Control}

In CDMA, the process of inner loop power control occurs as follows: In the reverse link direction (from MS to BS), the transmission power information goes to BS. At BS, the SIR target and the received SIR are calculated from the transmission power, the link-gain, and the noise power. Based on these factors, BS sends a TPC (Transmit-Power-Control) command to each MS at rate of $1,500 \mathrm{~Hz}$, or Sample Time $\left(T_{S}\right) \quad(=0.667 \mathrm{~ms})$ in the forward link direction (from BS to MS). This power equalization increases the maximum communication number between MSs and consequently eliminates the near-far effect.

\section{Review of Link-Budget Based SIR}

Changes in transmission power $[3,4,6]$ are represented in Eq. (1).

$$
P_{i}(t+1)=P_{i}(t)+\delta_{i}(t) \times T P C_{i}(t)
$$

where, $P_{i}(t)$ is the transmission power, $\delta_{i}(t)$ is the power control step-size, and $T P C_{i}(t)$ [6] is the TPC command for the $i$ th MS (MSi here) at time $t$.

$$
\begin{aligned}
& \operatorname{TPC}_{i}(t)=\operatorname{sign}\left(S I R_{\text {target }, i}(t)-S I R_{i}(t)\right) \times \\
& \left\lfloor\frac{\left.\log \left(1+\mu\left|x_{i}(t)\right|\right) / R_{P}\right)}{(1+\mu)}\left(2^{m}-1\right)\right\rfloor
\end{aligned}
$$

where, $\operatorname{SIR}_{\text {target }, i}(t)$ (= SIR target $_{(}(t)$ here) is the target SIR, $\operatorname{SIR}_{i}(t)$ is the received SIR from the $i$ th MS at time $t, \mu$ $=2^{(\mathrm{m}+1)}-1$ and $R_{p}$ is dynamic range of power adjustment. Non-uniform quantizer [12] used in voice coding is introduced that $(\mathrm{m}+1)$-bit TPC is adopted $T P C_{i}(t)=\left\{C_{0} C_{1} \ldots C_{m}\right\}$ where $C_{0}$ is the sign bit.

The paper [6] introduces the link-budget based SIR as

$$
S I R_{i}(t)=\frac{P_{R, i}(t)}{\sum_{j \neq i} P_{R, j i}(t)+P_{N}}
$$

where, $P_{R, i}$ is the received power from the $i$ th MS. $P_{R, j i}$ is the received power from the $j$ th MS with BS to which the $i$ th MS connects. The received power is affected by factors including the free space loss [13, 14] which has distance information and gaseous path loss [15] varied by humidity. The speed is estimated from moving distance per $T_{S}$ and $\operatorname{SIR}_{i}(t)$ is measured by distance variation.

The power delivered to the receiver of BS [6] is:

$$
\left.P_{R, i}=G_{T, i} \times P_{T, i} \times G_{R, i} / \mathbb{L}_{F}\left(D_{i}\right) \times L_{G}\left(D_{i}\right)\right)
$$

where, $G_{T, i}, P_{T, i}, G_{R, i}, L_{F}\left(D_{i}\right)$, and $L_{G}\left(D_{i}\right)$ are the transmission antenna gain, the transmission power, the received antenna gain of the $i$ th MS, the free space loss, and the gaseous path loss, respectively (the component loss is ignored here.). The speed is estimated from moving distance of free space loss. 
As MS1 moves away, the inner loop power control algorithms alter the transmission power to compensate for distance between $\mathrm{BS}$ and MS1. Eq. (5) [4, 6] measures the CTR as follows:

$$
\operatorname{CTR}(t)=\sum_{n=t-m+1}^{t} \frac{S F \times|\operatorname{TPC}(n)+\operatorname{TPC}(n-1)|}{m}
$$

where, $m=t$ if $t<w$, and $m=w$ if $t \geq w . w$ is the maximum size of the window average and $S F$ is scale factor.

There are several algorithms addressing inner loop power control in Fig. 2, speed and distance are estimated by CS-CLPC with the link-budget based SIR in this paper.

MS1 moves for $42,000 \times 0.667 \mathrm{~ms}$ (the number of the sample $=42,000$ ) at different speeds listed in Table 1.

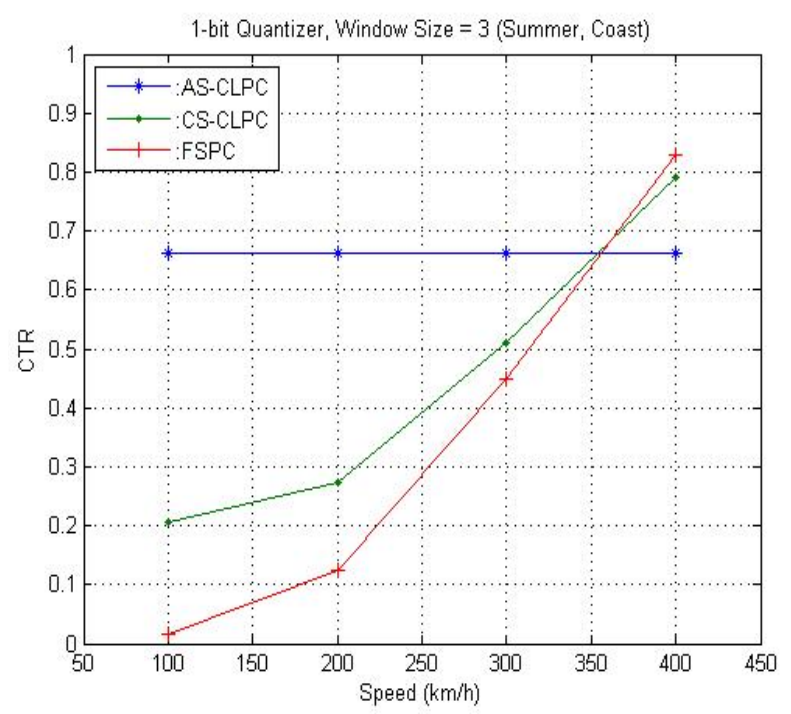

Fig. 2 CTR vs. MS1 speed at 1-bit Quantizer, window size 3 (Summer, Coast).

Table 1 MS1 moving distance for 28.0 sec $(=42,000 \times$ $0.667 \mathrm{~ms})$.

\begin{tabular}{lll}
\hline Speed $(\mathrm{km} / \mathrm{h})$ & Speed $(\mathrm{m} / \mathrm{s})$ & Moving distance $(\mathrm{m})$ \\
\hline 100 & 27.7778 & 0777.78 \\
200 & 55.5556 & $1,555.56$ \\
300 & 83.3333 & $2,333.33$ \\
400 & 111.1111 & $3,111.11$ \\
500 & 138.8889 & $3,888.89$ \\
600 & 166.6667 & $4,666.67$ \\
700 & 194.4436 & $5,444.42$ \\
800 & 222.2213 & $6,222.19$ \\
\hline
\end{tabular}

\section{Simulation Environments}

The frequency, the temperature, the pressure, and the bandwidth used here are set to $2.0 \mathrm{GHz}, 288 \mathrm{~K}$, $1,013 \mathrm{hPa}$, and $5 \mathrm{MHz}$, respectively. In Fig. 3, five MSs are arranged and $D_{i}$ s are set to $100 \mathrm{~m}, 200 \mathrm{~m}$, $300 \mathrm{~m}, 400 \mathrm{~m}$, and $500 \mathrm{~m}$. The antenna gain of each MS is set to $0 \mathrm{~dB}$, as is the antenna gain of BS. MS1 to MS5 complete their power control by FSPC, therefore each transmission power shown in Table 2 is different. Then, MS1 $500 \mathrm{~m}$ away from BS, starts to move. CTR is measured at different speeds.

BS which has one antenna can estimate MS distance only. But, BS which connects three RRHs can estimate distance and position together. In Fig. 1, RRH-A sends TPC to MS1 and measures CTR with (5). Then, from estimated speed with CTR, MS distance is calculated with respect to moving time. RRH-B, RRH-C cannot send TPC, but can inform RSSIs of MS1 for BS, then BS can estimate the position of MS1 with the ratio of these two RSSIs considering distance acquired by RRH-A. If RRH-B and RRH-C have same RSSI, MS1 is in the middle on red line of area2 in Fig. 1. If RRH-C does not get any radiation from MS1, it means MS1 is within area1,

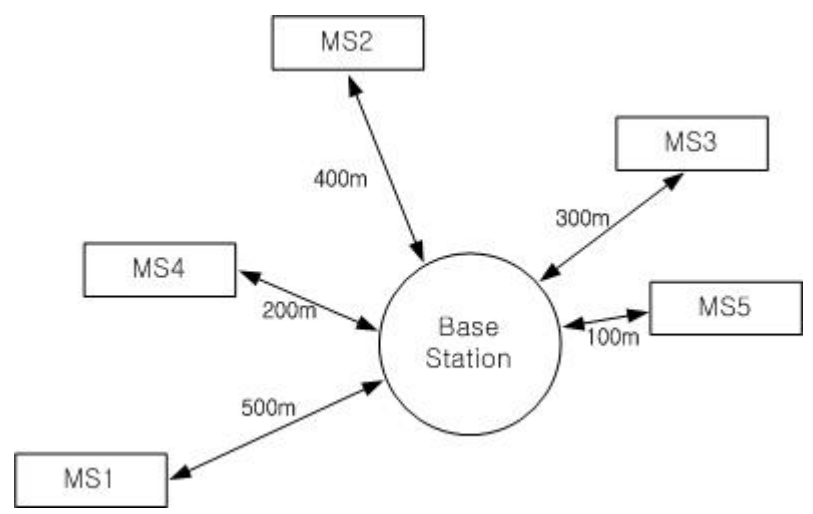

Fig. 3 Simulation formation.

Table 2 Initial condition of MS1 to MS5.

\begin{tabular}{lllll}
\hline & $\mathrm{P}(\mathrm{dB})$ & $\mathrm{G}(\mathrm{dB})$ & $\mathrm{G}(\mathrm{dB})$ & $\mathrm{D}(\mathrm{m})$ \\
\hline MS1 & +3.788 & 0.0 & 0.0 & 500 \\
MS2 & +1.850 & 0.0 & 0.0 & 400 \\
MS3 & -0.650 & 0.0 & 0.0 & 300 \\
MS4 & -4.168 & 0.0 & 0.0 & 200 \\
MS5 & -10.17 & 0.0 & 0.0 & 100 \\
\hline
\end{tabular}


vice versa in area3. As MS1 changes its RF power in every moment by power control, BS cares the RSSI ratio only. With this methodology, $\mathrm{BS}$ can track the MS distance and position.

\section{Simulation Results}

In Fig. 4, position (1) means RRH-B and RRH-C cannot measure RSSI of MS1, in other words, RF signal from MS1 cannot reach RRH-B or RRH-C, therefore MS1 is somewhere in area4. For the position (2), RF signal from MS1 cannot reach RRH-C so that MS1 is in area1. For the position (3), the ratio 4:6 means the ratio between lineB and lineC is 6:4 in Fig. 4 so that MS1 is placed toward RRH-C on the red line. Target position can be calculated only when MS is in area2. CTR measured from RRH-A is 0.27 and it is matching $200 \mathrm{~km} / \mathrm{h}$ in Fig. 2. This calculation is summarized in Table 3.

\section{Conclusion}

This work is motivated by the fact that MS speed has linearity with CTR calculated from adaptive power control algorithm. But MS position is unknown. This paper introduces that BS connecting three RRHs can estimate MS position with RSSI ratio together with MS distance using linearity between speed and CTR. One of RRHs provides CTR information for BS, the other two RRHs provide MS RSSI information for $\mathrm{BS}$, then BS calculates MS distance and position approximately with given RSSI ratio.

Future work might be related to distance and ratio error boundary due to timing error.

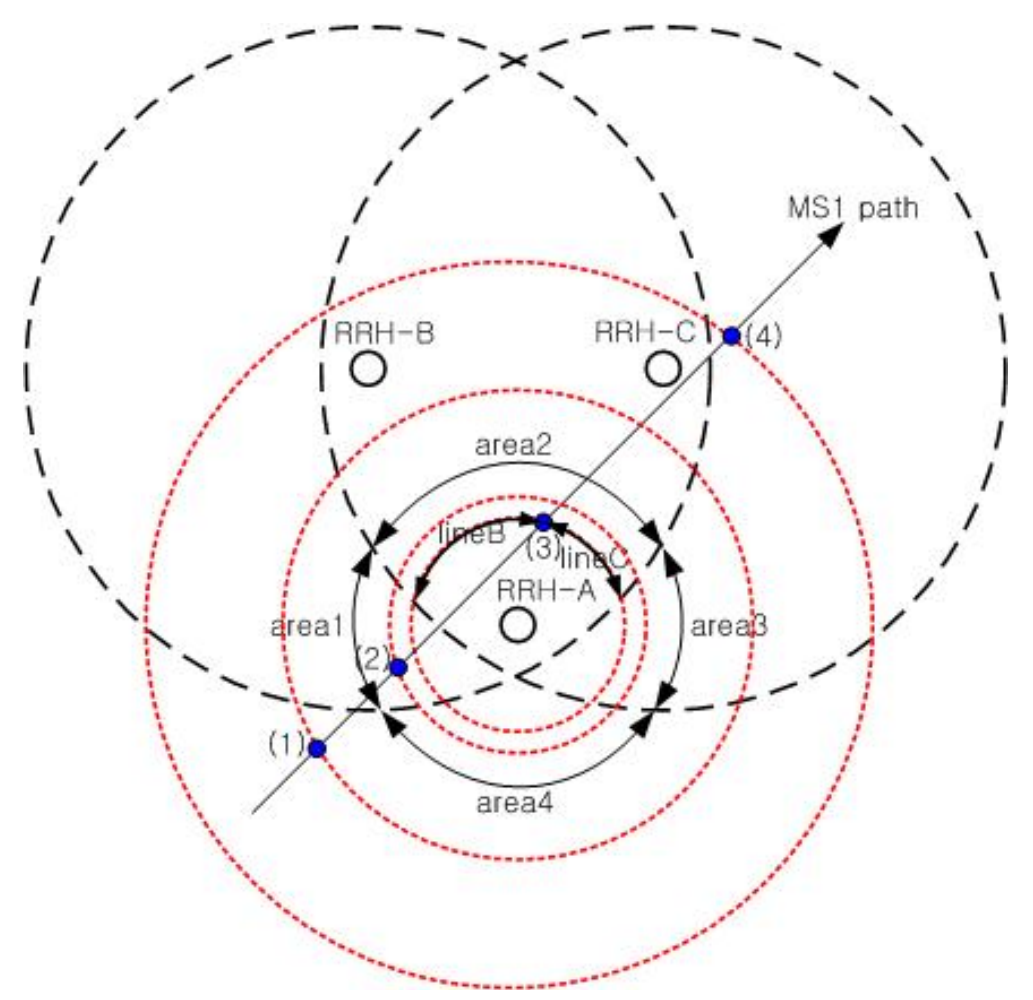

Fig. 4 Distance and position of MS1 according to MS path.

Table 3 The RSSI ratio between RRH-B and RRH-C and MS1 path.

\begin{tabular}{lllll}
\hline Position & CTR of CS = CLPC @RRH-A & $\begin{array}{l}\text { Time } \\
(\mathrm{sec})\end{array}$ & Moving Distance $(\mathrm{m})$ & RRSI (RRH-B: RRH-C) \\
\hline$(1)$ & $0.27(=200 \mathrm{~km} / \mathrm{h})$ & 0 & Origin $(500 \mathrm{~m}$ from BS) & Loss:loss \\
$(2)$ & $0.27(=200 \mathrm{~km} / \mathrm{h})$ & 5 & 277.78 & X:loss \\
$(3)$ & $0.27(=200 \mathrm{~km} / \mathrm{h})$ & 9 & 500.00 & $4: 6$ \\
$(4)$ & $0.27(=200 \mathrm{~km} / \mathrm{h})$ & 12 & 666.67 & Loss:X \\
\hline
\end{tabular}




\section{References}

[1] Perez-Romero, J., Sallent, O., Agusti, R., and Diaz-Guerra, M. A. 2005. Radio Resource Management Strategies in UMTS. NY, USA: John Wiley \& Sons.

[2] Ding, W., Wang, J., and Almagbile, A. 2010. "Adaptive Filter Design for UAV Navigation with GPS/INS/Optic Flow Integration." ICECE (Int. Conf. Electrical and Control Engineering), 4623-6.

[3] Patachaianand, R., and Sandrasegaran, K. 2007. "Performance Comparison of Adaptive Power Control in UMTS." Int. Conf. Wireless Broadband and Ultra Wideband Commun. (AusWireless 2007), 81-5.

[4] Patachaianand, R., and Sandrasegaran, K. 2007. "Consecutive Transmit Power Control Ratio Aided Adaptive Power Control for UMTS." Electronics Letters 43 (5): 55-6.

[5] Patachaianand, R., and Sandrasegaran, K. 2007. "User Speed Estimation Techniques for UMTS." Electronics Letters 43 (19): 1036-7.

[6] Lee, H.-C. 2011. "UAV speed estimation with multi-bit quantizer in Adaptive Power Control." The Seventh Int. Conf. Autonomic and Autonomous Systems (ICAS), 100-4.

[7] Lanzani,C. F., Kardaras, G., and Boppana, D. 2009.
"Remote Radio Heads the Evolution towards 4G Networks." Altera, Radiocomp Magazine.

[8] Kim, J. H., Lee, S. J., and Kim, Y. W. 1998. "Performance of Single-Bit Adaptive Step-Size Closed-Loop Power Control Scheme in DS-CDMA System." IEICE Trans. Commun. E81-B (7): 1548-52.

[9] Nourizadeh, S., Taaghol, P., and Tafazolli, R. 2000. "A Novel Closed-Loop Power Control for UMTS.” Int. Conf. $3 G$ Mobile Commun. Technologies (3G 2000), 56-9.

[10] Taaghol, P. 2004. "Speed-Adaptive Power Control for CDMA Systems." Bechtel Telecommunications Technical Journal 2 (1).

[11] Lee, H., and Cho, D. H. 2003. "A New User Mobility Based Adaptive Power Control in CDMA Systems." IEICE Trans. Commun. E86-B (5): 1702-5.

[12] Li, W., Dubey, V. K., and Law, C. L. 2001. "A New Generic Multistep Power Control Algorithm for the LEO Satellite Channel with High Dynamics." IEEE Commun. Lett. 5 (10): 399-401.

[13] Sklar, B. 1997. "Rayleigh Fading Channels in Mobile Digital Communication Systems Part I: Characterization." IEEE Communications Magazine: 90-100.

[14] Roddy, D. 1989. Satellite Communications. NJ, USA: Prentice Hall.

[15] ITU Recommendations. 2004. 